\title{
Simultaneous Wireless I nformation and Power Transfer in Two-hop OFDM Decode-and-Forward Relay Networks
}

\author{
Xiaofei $\mathrm{Di}^{1,2}$, Ke Xiong ${ }^{1,2}$, Yu Zhang ${ }^{3}$ and Zhengding Qiu ${ }^{1}$ \\ ${ }^{1}$ School of Computer and Information Technology, Beijing Jiaotong University, Beijing 100044, China \\ ${ }^{2}$ National Mobile Communications Research Laboratory, Southeast University, Nanjing 210018, China \\ ${ }^{3}$ School of Computer and Communication Engineering, University of Science and Technology Beijing, China \\ [e-mail: \{09112084, kxiong, zdqiu\}@bjtu.edu.cn, zhangyu.ustb.scce@foxmail.com] \\ *Corresponding author: Ke Xiong
}

Received August 19, 2015; revised October 28, 2015; accepted October 31, 2015; published January 31, 2016

\begin{abstract}
This paper investigates the simultaneous wireless information and power transfer (SWIPT) for two-hop orthogonal frequency division multiplexing (OFDM) decode-and-forward (DF) relay network, where a relay harvests energy from radio frequency signals transmitted by a source and then uses the harvested energy to assist information transmission from the source to its destination. The power splitting receiver is considered at the relay. To explore the performance limit of such a SWIPT-enabled system, a resource allocation (RA) optimization problem is formulated to maximize the achievable information rate of the system, where the power allocation, the subcarrier pairing and the power splitting factor are jointly optimized. As the problem is non-convex and there is no known solution method, we first decompose it into two separate subproblems and then design an efficient RA algorithm. Simulation results demonstrate that our proposed algorithm can achieve the maximum achievable rate of the system and also show that to achieve a better system performance, the relay node should be deployed near the source in the SWIPT-enabled two-hop OFDM DF relay system, which is very different from that in conventional non-SWIPT system where the relay should be deployed at the midpoint of the line between the source and the destination.
\end{abstract}

Keywords: Energy harvesting (EH), simultaneous wireless information and power transfer (SWIPT), relay network, orthogonal frequency division multiplexing (OFDM), resource allocation

This work was supported by the National Natural Science Foundation of China under Grants (no. 61201203) and also by the Open Research Fund of National Mobile Communications Research Laboratory, Southeast University (no. 2014D03). 


\section{Introduction}

\subsection{Background and Motivations}

Recently, relay communication has been widely investigated to improve the system capacity, reduce energy consumption and extend communication coverage [1]. In relay communication system, relay nodes are capable of employing various relaying protocols to assist to transmit information from a source to its destination. Among existing relaying protocols, amplify-and-forward (AF) and decode-and-forward (DF) are the two most popular ones [2, 3, 4].

Meanwhile, orthogonal frequency-division multiplexing (OFDM) has been adopted as the air interface in broadband wireless networks. By converting a frequency-selective wideband channel into a set of orthogonal narrowband frequency flat subcarrier channels, OFDM not only eliminates the intersymbol interference effectively, but also provides good design flexibility [5].

It is wildly believed that the combination of relaying and OFDM techniques is able to greatly improve the system performance [6]-[9]. For the OFDM relay system, resource allocation (RA) plays a very important role in system performance enhancement and thus requires to be effectively designed. For example, the incoming and outgoing subcarriers at the relay should be optimally paired over the two hops, which is well-known as subcarrier pairing (SP) and the available power at both the source and the relay nodes also should be optimally allocated over all subcarriers, which is power allocation (PA).

In some energy-constrained applications of OFDM relay system, such as wireless sensor networks (WSN) and wireless body area networks (WBAN), connecting the source or the relay to the power grid may be impossible. Although batteries can be deployed to solve this problem, the limited capacity and high transmit power may lead to quick depletion of the batteries. Therefore, the batteries are requested to be replaced or recharged frequently.

Recently, an alternative solution is proposed, i.e., to deploy energy harvesting (EH) technique in the systems, where $\mathrm{EH}$ nodes can harvest energy from their surrounding environment to maintain their operation. There are two different ways for communication nodes to harvest energy from surrounding physical space. One way is to harvest energy from solar, thermoelectric, and some other natural resources [10], but this way is heavily dependent on surrounding environment, which cannot supply continuous, reliable and steady energy source for systems. Another way is to harvest energy from radio frequency (RF) signals transmitted from the nodes with sufficient and stable energy supply, e.g., with power gird connections or high capacity batteries. More recently, this method is further evolved to realize the simultaneous wireless information and power transfer (SWIPT), due to the fact that RF signal carries both energy and information simultaneously.

However, to the best of our knowledge, SWIPT has not been fully studied in two-hop OFDM relay systems. Since OFDM has developed into a popular scheme for wideband digital communication and been used in applications such as digital television and audio broadcasting, DSL Internet access, wireless networks, powerline networks, and 4G mobile communications, it is significant to study SWIPT in two-hop OFDM relay systems. Moverover, as SP and PA at both source and the relay nodes play a very important role in system throughput enhancement for OFDM relay systems [6]-[9], in this paper, we shall jointly optimize them with SWIPT system factor for SWIPT-enabled OFDM DF relay systems. 


\subsection{Related work}

SWIPT has attracted much attention [11]-[25]. In the primary works (see e.g. [11] and [12]), an ideal receiver was assumed so that information decoding (ID) and EH can be simultaneously performed with the same received signals in SWIPT-enabled systems. Later, this assumption was considered to be impractical for real wireless systems, since circuits for harvesting energy from RF signals cannot be used to decode the carried information directly [13]. Hence, two practical SWIPT receiver architectures, time switching (TS) and power splitting (PS), were also proposed in [13]. When TS is applied, the received signal is either processed by an energy receiver for $\mathrm{EH}$ or processed by an information receiver for ID from the perspective of time. When PS is applied, the received signal is split into two power streams by a power splitter, with one stream to the energy receiver and the other one to the information receiver.

These two practical SWIPT receiver architectures thus far have been widely investigated. In [14], the TS and PS architectures with PA were studied to explore the capacity-energy tradeoff in point-to-point channels. Later, both architectures were extended to multi-subcarrier OFDM systems, see e.g., [15]-[17], where the system throughput, energy efficiency and weighted sum rate were optimized, respectively. Besides the work on SWIPT-enabled single-hop communication systems [14]-[17], SWIPT in two-hop relay systems has also attracted more and more attention, see e.g., [18]-[22]. In [18], outage probability was analyzed for two-hop one way AF relay-aided systems where both TS and PS reciever architectures were considered. The outage performances were respectively studied for two-way relay system with TS receiver architecture in $[19,20]$ and for relay broadcast system with TS and PS receiver architectures in [21]. In [22], joint information and energy cooperation was studied for cognitive radio networks with PS and TS reciever architectures, where the cooperation between the primary and secondary systems was involved. However, all the works on relay systems metioned above were done for single carrier wireless systems.

Lately, a few works began to investigate SWIPT in two-hop OFDM relay networks. In [23], the achievable information rate was maximized for two-hop OFDM relay systems with TS and PS reciever architectures, where the SP, the PS factor and the PA at both source and the relay were jointly optimized but only AF relaying protocol was involved. In [24] and [25], SWIPT was investigated in two-hop DF OFDM relay networks with PS receiver architecture, where the achievable information rate was maximized by joint optmization of PS and PA. However, in [24], PA was only performed at the source and the harvested energy at the relay was not re-allocated, and in [24, 25], the SP over the two hops was not involved.

\subsection{Contributions}

In this paper, we also study the SWIPT in two-hop OFDM relay systems, in which the SP over the two hops, the PA at both the source and the relay nodes and the PS factor are jointly optimally designed. We consider the SWIPT in the three-node two-hop OFDM DF relay system, where a source communicates with its destination via a half-duplex DF relay. It is assumed that the source has fixed energy supply but the relay has no fixed energy supply. The relay is an EH node and needs to harvest energy from RF signal transmitted from the source, and then, it can help the information transmission between the source and the destination with its harvested energy. The PS receiver architecture is considered at the relay.

Our main contributions are summarized as follows. Firstly, in order to explore the system performance limit for such a SWIPT-enabled OFDM DF relay system, we formulate a RA optimization problem to maximize the achievable information rate for it. Different from the existing similar work, i.e., [24, 25], in our RA scheme design, the SP, the PS factor and the PA 
at both source and the relay are jointly optimized, and the total harvested energy at the relay over all subcarriers is re-allocated for the second hop. Secondly, since the optimization problem is non-convex and doesn't have known solution method, we design a low-complex efficient RA algorithm for it by decomposing it into two separate subproblems. Specifically, for the optimal SP, the incoming and outgoing subcarriers are paired at the relay according to two hops channel gains. With the optimal SP, for the optimal PA and PS factor, a joint optimization method is proposed to obtain them. Thirdly, extensive simulation experiments are performed to evaluate the system performance. The results demonstrate that by using our proposed RA algorithm, the maximal achievable information rate of the system can be reached. It is also shown that, with the same total available power consumption, the SWIPT-enabled OFDM DF relay system has some performance loss compared with the conventional non-SWIPT OFDM DF relay system. Moreover, the SWIPT-enabled system shows very different behavior from the conventional non-SWIPT system w.r.t the relay position. That is, to obtain a better system performance, for the SWIPT-enabled system, the relay should be deployed near the source, but for the non-SWIPT system, it should be deployed near the middle point of the line between the source and the destination. These may provide some useful insights for the understanding and designing of SWIPT-enabled relay systems.

\subsection{Organization}

This paper is organized as follows. In Section 2, the system model is presented and then a RA optimization problem is formulated. The solution of the RA optimization problem is given in Section 3. Simulation results are provided in Section 4 for performance evaluations and comparisons. Finally, Section 5 summarizes this paper.

\section{System Model and Problem Formulation}

\subsection{System Model}

Consider a two-hop OFDM relay system, which consists of one source, one destination and one relay, as shown in Fig. 1. The source desires to transmit information to the destination with the assistance of the relay, and it is assumed that there is no direct link between the source and the destination. The relay operates in half-duplex mode, so that it cannot simultaneously transmit and receive signals. The source is with fixed power supply, and its available power is denoted by $P_{S}$. The relay is an energy harvesting (EH) node with DF relaying protocol employed, so it harvests energy from the RF signals transmitted from the source and then uses the harvested energy to help to forward the decoded information from the source to its destination.

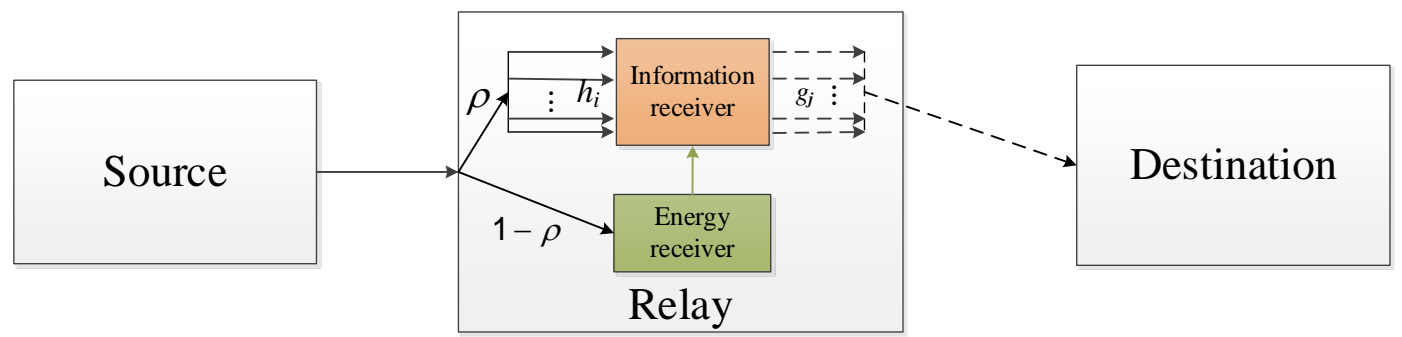

Fig. 1. SWIPT-enabled two-hop OFDM DF relay system where one relay harvests energy transmitted by one source and then help to transmit information from the source to its destination. 
For such a SWIPT-enabled system, the two-hop transmission is on a time-frame basis with each frame consisting of multiple OFDM symbols. Each frame is of length $T$, which is further divided into two equal time slots.

In the first time slot, the source transmits OFDM symbols to the relay. Thus, the received signal at the relay over subcarrier $i$ can be given by

$$
y_{r, i}=\sqrt{P_{s, i}} h_{i} x_{i}+z_{r, i}, \forall i \in\{1, \ldots, N\},
$$

where $N$ is the number of subcarriers of the system, $x_{i}$ and $h_{i}$ denote the transmitted symbol and the fading coefficient over subcarrier $i$, respectively. $z_{r, i}$ represents the additive white Gaussian noise (AWGN) with zero mean and variance $\sigma_{r \text {,att }}^{2}$ from the antenna over subcarrier $i$ at the relay. $P_{s, i}$ denotes the transmit power at the source over subcarrier $i$, which satisfies that

$$
\sum_{i=1}^{N} P_{s, i} \leq P_{S} .
$$

The PS receiver architecture is adopted at the relay so that it can split the received RF signals into two power streams to perform EH and ID, respectively. Let $\rho$ be the PS ratio which is the fraction of the signal power used for ID, satisfying that

$$
0 \leq \rho \leq 1 \text {. }
$$

Thus, the rest $1-\rho$ part of the power is used for EH. The energy is directly harvested from the received RF signals over all subcarriers. For practical implementation, all subcarriers are assumed to share the identical PS ratio $\rho$ [15], and thus the total harvested energy at the relay can be given by

$$
E_{R}=\frac{T}{2} \eta(1-\rho) \sum_{i=1}^{N}\left|h_{i}\right|^{2} P_{s, i},
$$

where $\eta$ is a constant, denoting the energy harvesting efficiency and satisfying $0 \leq \eta \leq 1$. As a result, the total available power at the relay is

$$
P_{R}=\frac{E_{R}}{T / 2}=\eta(1-\rho) \sum_{i=1}^{N}\left|h_{i}\right|^{2} P_{s, i} .
$$

In the second time slot, with the power stream for ID, the relay decodes the received symbols and then forward the re-encoded symbols to the destination by consuming the harvested energy. Subcarrier pairing is considered, so the information received over subcarrier $i$ of the first hop can be forwarded over the subcarrier $j$ of the second hop. Thus the received signal over subcarrier $j$ received at the destination can be given by

$$
y_{d, j}=\sqrt{P_{r, j}} g_{j} x_{i}+z_{d, j}, \forall j \in\{1, \ldots, N\},
$$

where $g_{j}$ is fading coefficient over subcarrier $j$ at the destination, $z_{d, j}$ is AWGN with zero mean and variance $\sigma_{d \text {,att }}^{2}$ from the antenna over subcarrier $j$ at the destination, and $P_{r, j}$ is the available power over subcarrier $j$ at the relay and satisfies that $\sum_{j=1}^{N} P_{r, j} \leq P_{R}$, i.e.,

$$
\sum_{j=1}^{N} P_{r, j} \leq \eta(1-\rho) \sum_{i=1}^{N}\left|h_{i}\right|^{2} P_{s, i} .
$$

\subsection{Optimization Problem Formulation}

In order to explore the performance limit of the presented SWIPT-enabled OFDM DF relay system, in this subsection we formulate an optimization problem to maximize the achievable information rate of the system.

As is known, the achievable information rate between the source and its destination for DF 
relay system over a subcarrier pair $(i, j)$ is limited by the minimum rate over the two hops [2], which can be given by

$$
R_{i, j}=\frac{1}{2} \min \left\{\log _{2}\left(1+\frac{\rho\left|h_{i}\right|^{2} P_{s, i}}{\rho \sigma_{r, \text { att }}^{2}+\sigma_{r, \text { proc }}^{2}}\right), \log _{2}\left(1+\frac{\left|g_{j}\right|^{2} P_{r, j}}{\sigma_{d, \text { att }}^{2}+\sigma_{d, \text { proc }}^{2}}\right)\right\},
$$

where $\sigma_{r, \text { proc }}^{2}$ and $\sigma_{d, \text { proc }}^{2}$ denote the signal processing noise power over each subcarrier at the relay and the destination, respectively [16]. For convenience, let $\sigma_{d}^{2}=\sigma_{d \text {,att }}^{2}+\sigma_{d \text {,proc }}^{2}$, which denotes the total noise power received at the destination over each subcarrier. The first term $\log _{2}\left(1+\frac{\rho\left|h_{i}\right|^{2} P_{s, i}}{\rho \sigma_{r, \text { att }}^{2}+\sigma_{r, \text { proc }}^{2}}\right)$ in (4) is the mutual information from the source to the relay over subcarrier $i$ [16], and the second term $\log _{2}\left(1+\frac{\left|g_{j}\right|^{2} P_{r, j}}{\sigma_{d, \text { att }}^{2}+\sigma_{d, \text { proc }}^{2}}\right)$ in (4) is the mutual information from the relay to the destination over subcarrier $j$. The pre-min factor $1 / 2$ is due to two time slots in each frame.

Hence, the achievable information rate of the system can be defined as

$$
R(\mathrm{P}, \mathrm{S}, \rho)=\sum_{i=1}^{N} \sum_{j=1}^{N} s_{i, j} R_{i, j},
$$

where $\mathrm{P}=\left\{P_{s, i} \geq 0, P_{r, j} \geq 0 \mid \forall i, j\right\}$ represents the PA policy and $\mathrm{S}=\left\{s_{i, j} \in\{0,1\} \mid \forall i, j\right\}$ denotes the SP policy, which indicates that if the incoming subcarrier $i$ is paired with the outgoing subcarrier $j, s_{i, j}=1$. Otherwise, $s_{i, j}=0$. Moreover, one incoming subcarrier is only allowed to be paired with one outgoing subcarrier and vice versa. Thus,

$$
\sum_{j=1}^{N} s_{i, j} \leq 1, \forall i, \sum_{i=1}^{N} s_{i, j} \leq 1, \forall j .
$$

As a result, the optimization problem can be formulated as (P1)

$$
\begin{aligned}
& \max _{\mathrm{P}, \mathrm{S}, \rho} R(\mathrm{P}, \mathrm{S}, \rho)=\sum_{i=1}^{N} \sum_{j=1}^{N} \mathrm{~s}_{i, j} R_{i, j} \\
& \text { s.t. (1),(2),(3),(5). }
\end{aligned}
$$

It can be observed that the problem $\mathbf{P 1}$ is a mixed integer programming problem and its objective function and constraint (3) imply a complicated coupling among the source PA, the relay PA and the PS ratio, which makes it difficult to deal with by using traditional methods. Therefore, in the next section, we shall design an efficient joint RA algorithm to solve it.

\section{Problem Solution}

This section shall describe our proposed RA algorithm to solve Problem P1 at first and then analyze its computational complexity.

\subsection{Our Proposed RA Algorithm}

The framework of our proposed solution for Problem P1 is shown in Algorithm 1 (RA algorithm), which contains two separate steps. In Step 1), the optimal SP is obtained, and in Step 2), the joint optimal PS and PA are obtained. The detailed process of each step is 
described as the two following subsectons. Specifically, the optimal SP is obtained in subsection 3.1.1, and the joint optimal PS and PA are obtained in subsection 3.1.2.

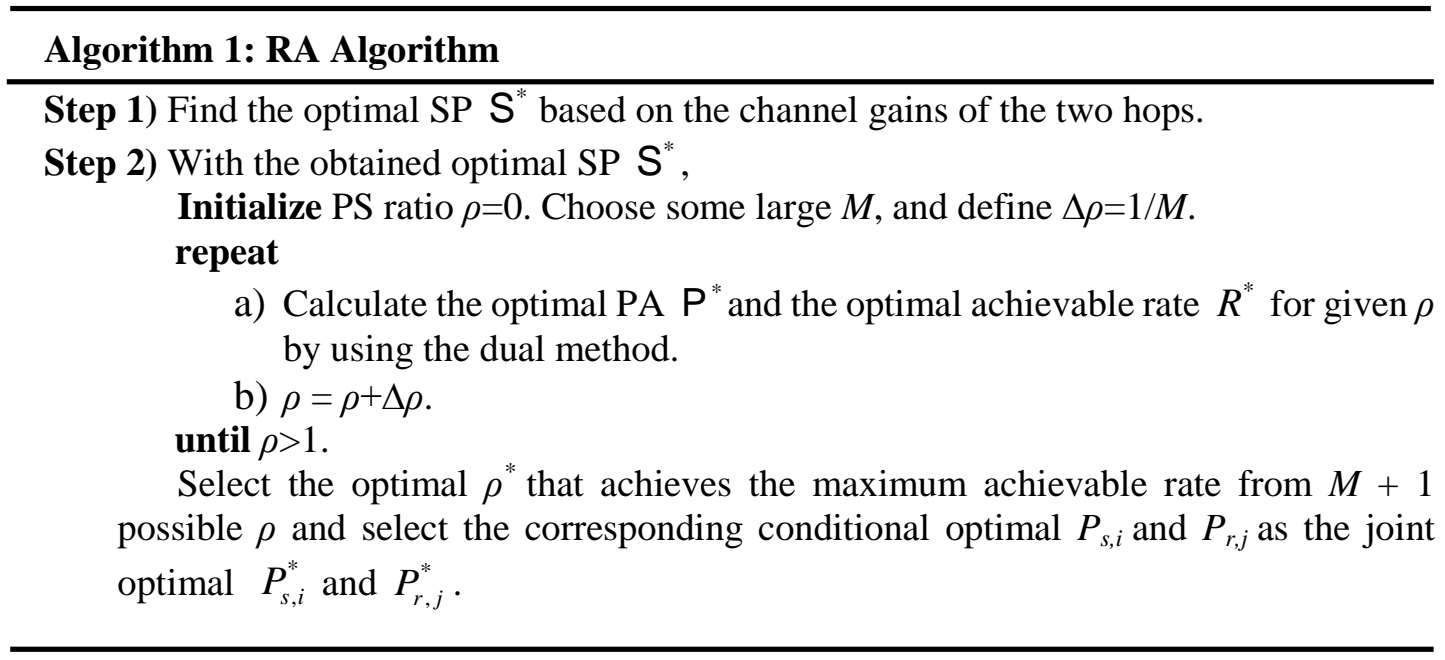

\subsubsection{Obtain the optimal SP $\mathrm{S}^{*}$}

The proposed SP scheme is performed on the channel gains of two hops as follows. First, both the incoming subcarriers and the outgoing subcarriers are sorted from highest to lowest according to the values of the channel gains $\left|h_{i}\right|^{2}$ and $\left|g_{j}\right|^{2}$ over the two hops, respectively. Then, the $k$-th incoming subcarrier is paired with the $k$-th outgoing subcarrier, where $k \in\{1, \ldots, N\}$. This scheme is referred to as the CG-sorted pairing in the sequel. This scheme is optimal and the result is given in the following lemma.

Lemma 1: The CG-sorted pairing scheme in Algorithm 1 is optimal for the problem $\mathbf{P 1}$.

Proof: In the proposition 3 of [26], it was proved that the optimal subcarrier pairing scheme can be achieved by the signal-to-noise ratio (SNR)-sorted scheme. This scheme is also applicable for the presented SWIPT system, and this can be verified by the silimar method in [26]. In our presented CG-sorted scheme, the subcarrier pairing is performed on the sorted channel gains. But, we can prove that in our considered SWPIT system, the proposed CG-sorted scheme always leads to the same sorted result as the SNR-sorted method. Thus, our presented CG-sorted scheme achieves the optimum. This can be explained as follow. Let $Q_{i}=\frac{\rho\left|h_{i}\right|^{2}}{\rho \sigma_{r, \text { att }}^{2}+\sigma_{r, \text { proc }}^{2}}, \quad M_{j}=\frac{\left|g_{j}\right|^{2}}{\sigma_{d}^{2}}$, and $U_{i}=Q_{i} P_{s, i}$, where $U_{i}$ represents the received SNR over subcarrier $i$ of the first hop. Assuming two subcarriers $i_{1}$ and $i_{2}$ satisfy $\left|h_{i_{1}}\right|^{2}>\left|h_{i_{2}}\right|^{2}$, it can easily be proved that $Q_{i_{1}}>Q_{i_{2}}$. Obviously, when the system achieves the optimality, all the power at the source should be depleted. By using the property and the similar method in the proposition 4 of [26], we can obtain that $U_{i_{1}}>U_{i_{2}}$. This means that a better channel gain provides a higher SNR. For the second hop, a similar result also can be obtained. Thus, Lemma 1 is proved.

Since the subcarrier pairing is only dependent on channel gains and is not related to PA and PS ratio $\rho$, Algorithm 1 can be divided into two separate steps and still maintain the optimality. 


\subsubsection{Obtain the optimal $\rho^{*}$ and the optimal $\mathrm{P}^{*}$ with the obtained $\mathrm{S}^{*}$}

In Step 1), the optimal $S^{*}$ is obtained. Denote the obtained subcarrier-pair set to be $S P=\left\{(i, j) \mid s_{i, j}=1, \forall i, j\right\}$. By using the optimal $S^{*}$ and introducing a set $\mathrm{R}=\left\{R_{i, j} \mid \forall(i, j) \in S P\right\}$ and considering (4), Problem P1 is equivalently transformed to be the following problem (P2)

$$
\begin{array}{ll}
\min _{\mathrm{P}, \rho, \mathrm{R}}- & \sum_{(i, j) \in \mathrm{SP}} R_{i, j} \\
\text { s.t. } \quad(1),(2),(3), & \\
& R_{i, j}-\frac{1}{2} \log _{2}\left(1+\frac{\rho\left|h_{i}\right|^{2} P_{s, i}}{\rho \sigma_{r, \text { att }}^{2}+\sigma_{r, \text { proc }}^{2}}\right) \leq 0, \forall(i, j) \in S P, \\
& R_{i, j}-\frac{1}{2} \log _{2}\left(1+\frac{\left|g_{j}\right|^{2} P_{r, j}}{\sigma_{d}^{2}}\right) \leq 0, \forall(i, j) \in S P .
\end{array}
$$

It can be seen that the objective function is convex, and the constraints (1), (2) and (7) are convex set of the optimized variables. However, Problem P2 is still non-convex due to the constraints (3) and (6). Fortunately, we find that for a given $\rho$, the constraint (3) is linear to $P_{s, i}$ and $P_{r, j}$, and the constraint (6) becomes a convex set of the optimized variables. In this case, Problem P2 becomes a convex optimization problem. This motivates us to first solve the convex optimization problem to get the conditional optimal $P_{s, i}$ and $P_{r, j}$ for a given $\rho$, and then find the optimal PS ratio $\rho$.

Firstly, for a given $\rho$, to better understand the system and get some explicit results on the conditional optimal $P_{s, i}$ and $P_{r, j}$, a dual method is adopted to solve the convex optimization problem, as shown in Step 2-a) of Algorithm 1. The detailed operations of Step 2-a) are explained as follows.

With a given PS ratio $\rho$, the optimal PA $\mathbf{P}^{*}$ is derived via solving the dual problem of $\mathbf{P} 2$. The Lagrangian of $\mathbf{P} \mathbf{2}$ is given by

$$
\begin{aligned}
& \mathrm{L}(\boldsymbol{\alpha}, \boldsymbol{\beta}, \lambda, \gamma, \mathrm{P}, \mathrm{R})=-\sum_{(i, j) \in S P} R_{i, j} \\
& +\sum_{(i, j) \in S P} \alpha_{i, j}\left(R_{i, j}-\frac{1}{2} \log _{2}\left(1+Q_{i} P_{s, i}\right)\right)+\sum_{(i, j) \in S P} \beta_{i, j}\left(R_{i, j}-\frac{1}{2} \log _{2}\left(1+M_{j} P_{r, j}\right)\right) \\
& +\lambda\left(\sum_{i=1}^{N} P_{s, i}-P_{S}\right)+\gamma\left(\sum_{j=1}^{N} P_{r, j}-\eta(1-\rho) \sum_{i=1}^{N}\left|h_{i}\right|^{2} P_{s, i}\right),
\end{aligned}
$$

where $\lambda \geq 0, \gamma \geq 0$ are two Lagrange multipliers and $\boldsymbol{\alpha}$ and $\boldsymbol{\beta}$ are two Lagrange multiplier vectors composed of the elements $\alpha_{i, j} \geq 0, \forall(i, j) \in S P$ and $\beta_{i, j} \geq 0, \forall(i, j) \in S P$, respectively.

The dual function is then defined as $g(\boldsymbol{\alpha}, \boldsymbol{\beta}, \lambda, \gamma)=\min _{\mathrm{P}, \mathrm{R}} \mathrm{L}(\boldsymbol{\alpha}, \boldsymbol{\beta}, \lambda, \gamma, \mathrm{P}, \mathrm{R})$, and the dual problem is given by $\max _{\boldsymbol{\alpha}, \boldsymbol{\beta}, \lambda, \gamma} g(\boldsymbol{\alpha}, \boldsymbol{\beta}, \lambda, \gamma)$. To obtain $g(\boldsymbol{\alpha}, \boldsymbol{\beta}, \lambda, \gamma)$, we first apply Karush-Kuhn-Tucker (KKT) conditions to calculate the optimal $\mathbf{P}^{\#}$ for given $\boldsymbol{\alpha}, \boldsymbol{\beta}, \lambda, \gamma$. With the derivation on (8) w.r.t $R_{i, j}$ equal to zero, we can get that $\alpha_{i, j}+\beta_{i, j}=1, \forall(i, j) \in \mathrm{SP}$. Thus, $\beta_{i, j}$ in $g(\boldsymbol{\alpha}, \boldsymbol{\beta}, \lambda, \gamma)$ is substituted by $\beta_{i, j}=1-\alpha_{i, j}$. As a result, $g(\boldsymbol{\alpha}, \boldsymbol{\beta}, \lambda, \gamma)$ is reduced to $g(\boldsymbol{\alpha}, \lambda, \gamma)=\min _{\mathrm{P}} \mathrm{L}(\boldsymbol{\alpha}, \lambda, \gamma, \mathrm{P})$, where 


$$
\begin{aligned}
& \mathrm{L}(\boldsymbol{\alpha}, \lambda, \gamma, \mathrm{P})= \\
& -\sum_{(i, j) \in \mathrm{SP}} \frac{\alpha_{i, j}}{2} \log _{2}\left(1+Q_{i} P_{s, i}\right)-\sum_{(i, j) \in \mathrm{SP}} \frac{1-\alpha_{i, j}}{2} \log _{2}\left(1+M_{j} P_{r, j}\right) \\
& +\lambda\left(\sum_{i=1}^{N} P_{s, i}-P_{S}\right)+\gamma\left(\sum_{j=1}^{N} P_{r, j}-\eta(1-\rho) \sum_{i=1}^{N}\left|h_{i}\right|^{2} P_{s, i}\right) .
\end{aligned}
$$

Further, by letting the derivations on (9) w.r.t $P_{s, i}$ and $P_{r, j}$ equal to zero, we can obtain the optimal $P_{s, i}$ and $P_{r, j}$ for given $\boldsymbol{\alpha}, \lambda, \gamma$ as

$$
\begin{gathered}
P_{s, i}^{\#}=\left[\frac{\alpha_{i, j} / 2}{\left(\lambda-\gamma \eta(1-\rho)\left|h_{i}\right|^{2}\right) \ln (2)}-\frac{1}{Q_{i}}\right]^{+}, \\
P_{r, j}^{\#}=\left[\frac{\left(1-\alpha_{i, j}\right) / 2}{\gamma \ln (2)}-\frac{1}{M_{j}}\right]^{+},
\end{gathered}
$$

where $[x]^{+}=\max (x, 0)$. Note that if $\lambda-\gamma \eta(1-\rho)\left|h_{i}\right|^{2}<0$, the derivation on (9) w.r.t $P_{s, i}$ is less than zero, i.e., $\mathrm{L}(\boldsymbol{\alpha}, \lambda, \gamma, \mathrm{P})$ is a decreasing function of $P_{s, i}$, then the optimal $P_{s, i}$ is $P_{s, i}^{\#}=P_{S}$.

Substituting $P_{s, i}^{\#}$ and $P_{r, j}^{\#}$ into (9), we obtain the conditioned $g(\boldsymbol{\alpha}, \lambda, \gamma)$ on $\mathrm{P}^{\#}$.

Since a dual function is always convex by definition, we solve the dual problem by using the gradient-based method. The subgradients of $\lambda, \gamma$ and $\alpha_{i, j}$ are respectively chosen as

$$
\begin{gathered}
\Delta \lambda=\sum_{i=1}^{N} P_{s, i}^{\#}-P_{S}, \\
\Delta \gamma=\sum_{j=1}^{N} P_{r, j}^{\#}-\eta(1-\rho) \sum_{i=1}^{N}\left|h_{i}\right|^{2} P_{s, i}^{\#}, \\
\Delta \alpha_{i, j}=\frac{1}{2} \log _{2}\left(1+M_{j} P_{r, j}^{\#}\right)-\frac{1}{2} \log _{2}\left(1+Q_{i} P_{s, i}^{\#}\right), \forall(i, j) \in S P .
\end{gathered}
$$

Once the gradient-based iteration converges, the final optimal $\mathrm{P}^{*}$ and the optimal achievable rate $R^{*}$ are obtained for a given $\rho$.

Secondly, we try to find the optimal PS ratio $\rho^{*}$. Obviously, the obtained optimal $\mathrm{P}^{*}$ and the obtained optimal achievable rate $R^{*}$ above are a function of the PS ratio $\rho$, which turn out to be neither convex nor concave. As the closed-form solution of the PS ratio $\rho$ seems infeasible, we resort to a numerical method, i.e., by linearly searching all possible $\rho$ with a sufficiently small step-size $\Delta \rho$, and selecting the one with the maximum achievable information rate as the final optimal $\rho^{*}$ and the corresponding conditional optimal $P_{s, i}$ and $P_{r, j}$ as the joint optimal $P_{s, i}^{*}$ and $P_{r, j}^{*}$. The complete procedure is shown in Step 2 of Algorithm 1.

\subsection{Computational Complexity Analysis}

Now, we analyze the computational complexity of Algorithm 1. The complexity of Step 1 of Algorithm 1 depends on the adopted sorting method, which is $O(N \log N)$ if the quick-sort method is applied. Moreover, the complexity of the Step 2-a) is $O\left(N(N+2)^{q}\right)$ (if the ellipsoid method is adopted, $q=2$ ), and the complexity searching optimal $\rho^{*}$ is $O(M)$. Thus, the total complexity can be expressed by $O\left(N \log N+M N(N+2)^{q}\right)$. For comparison, the complexity of a benchmark method which adopts the exhaustive computer search for SP and the same Step 
2 of Algorithm 1 for PA and PS is $O\left(N ! M N(N+2)^{q}\right)$, which is much higher than our proposed Algorithm 1.

\section{Simulation Results}

This section provides some simulation results to discuss the performance of our proposed RA algorithm. To figure out the system performance of the SWPIT-enabled OFDM DF relay system, the performance of the conventional non-SWIPT OFDM DF relay system is also simulated. In our simulations, $\eta$ is set to be 1 , which has been widely adopted to explore the performance limit of SWIPT-enabled systems. $\sigma_{r, \text { att }}^{2}, \sigma_{r, \text { proc }}^{2}$ are set to be $-33 \mathrm{dBm}$ and $\sigma_{d}^{2}$ is $-30 \mathrm{dBm}$ [27]. It is assumed that all the three nodes are located on a same line. The distance between the source and the destination is denoted by $d_{0}$ as a reference distance, where $d_{0}$ is set to be $10 \mathrm{~m}$. The position of the relay is described by the normalized distance $d_{r} / d_{0}$, where $d_{r}$ represents the distance between source and relay.

Channel coefficients $h_{i}$ and $g_{j}$ are picked from a Rayleigh fading channel with the distribution [28] as

$$
h_{i} \sim \mathrm{ON}\left(0, \frac{1}{L\left(1+d_{r}\right)^{\alpha}}\right)
$$

and

$$
g_{j} \sim \mathrm{ON}\left(0, \frac{1}{L\left(1+\left(d_{0}-d_{r}\right)\right)^{\alpha}}\right),
$$

respectively, where the path loss exponent $\alpha=3$ and the number of channel taps is set to $L=4$.

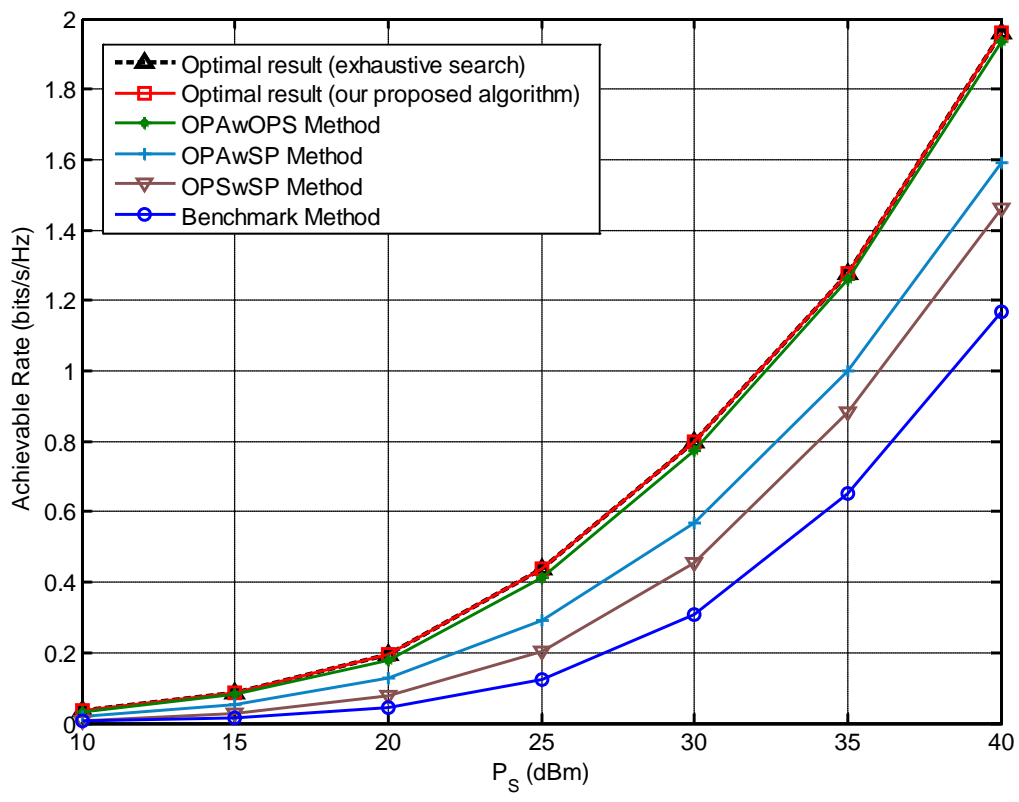

Fig. 2. Achievable rate versus $P_{S}$ with $N=4$ and the relay located at the middle point between the source and its destination. 


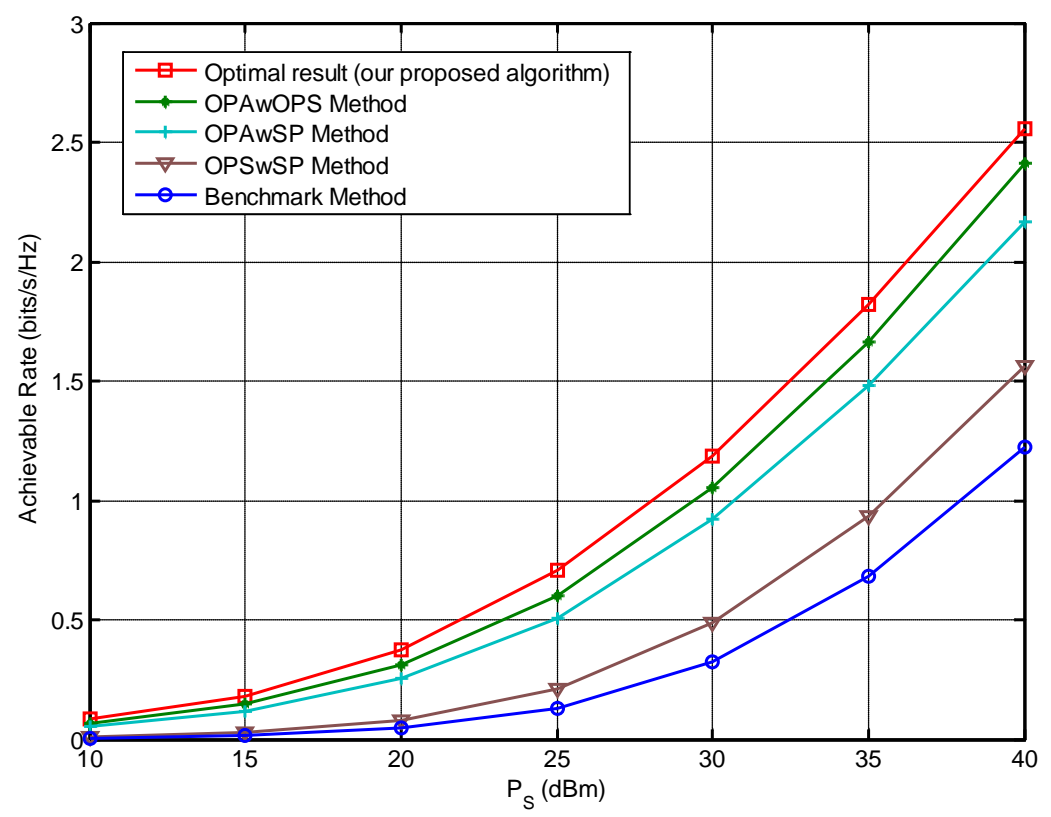

Fig. 3. Achievable rate versus $P_{S}$ with $N=64$ and the relay located at the middle point between the source and its destination.

Firstly, to show the performance of our proposed RA algorithm, four other methods are also simulated and compared, i.e., 1)Benchmark Method: Uniform PA and fixed $\rho=0.5$ without SP; 2)OPSwSP Method: Uniform PA and optimal $\rho$ with SP; 3)OPAwSP Method: Optimal PA and fixed $\rho=0.5$ with SP; 4)OPAwOPS Method: Optimal PA and optimal $\rho$ without SP. Fig. 2 and Fig. 3 compare the achievable information rates of the five algorithms versus the power $P_{S}$ for $N=4$ and $N=64$, respectively. From these two figures, it is observed that our proposed algorithm is superior to the other four algorithms. In Fig. 2, the optimal RA achieved by exhaustive search is also plotted. It shows that our proposed algorithm achieves the same performance with optimal RA achieved by exhaustive search, which implies that our proposed algorithm is also the optimal RA for the SWIPT-enabled OFDM DF relay system.

Now, we compare the achievable information rate of the SWIPT-enabled OFDM DF relay system with that of conventional non-SWIPT OFDM DF relay system. For the non-SWIPT system, let $\tilde{P}_{s, i}$ be the power over subcarrier $i$ at the source and $\tilde{P}_{r, i}$ be the power over subcarrier $i$ at the relay, so the consumed energy over $T$ is $\tilde{E}=\frac{T}{2} \sum_{i=1}^{N} \tilde{P}_{s, i}+\frac{T}{2} \sum_{i=1}^{N} \tilde{P}_{r, i}$. For the SWIPT-enabled system, the consumed energy over $T$ is $E=\frac{T}{2} \sum_{i=1}^{N} P_{s, i} \leq \frac{T}{2} P_{S}$. To guarantee the comparison fairness, it requires that $\tilde{E}=E$. So, for the non-SWIPT system, it satisfies the total power constraint $\sum_{i=1}^{N} \tilde{P}_{s, i}+\sum_{i=1}^{N} \tilde{P}_{r, i} \leq P_{S}$. With this constraint, to simulate the achievable information rate of the non-SWIPT OFDM DF relay system, we performed the optimal RA for it by using the method proposed in [7]. 


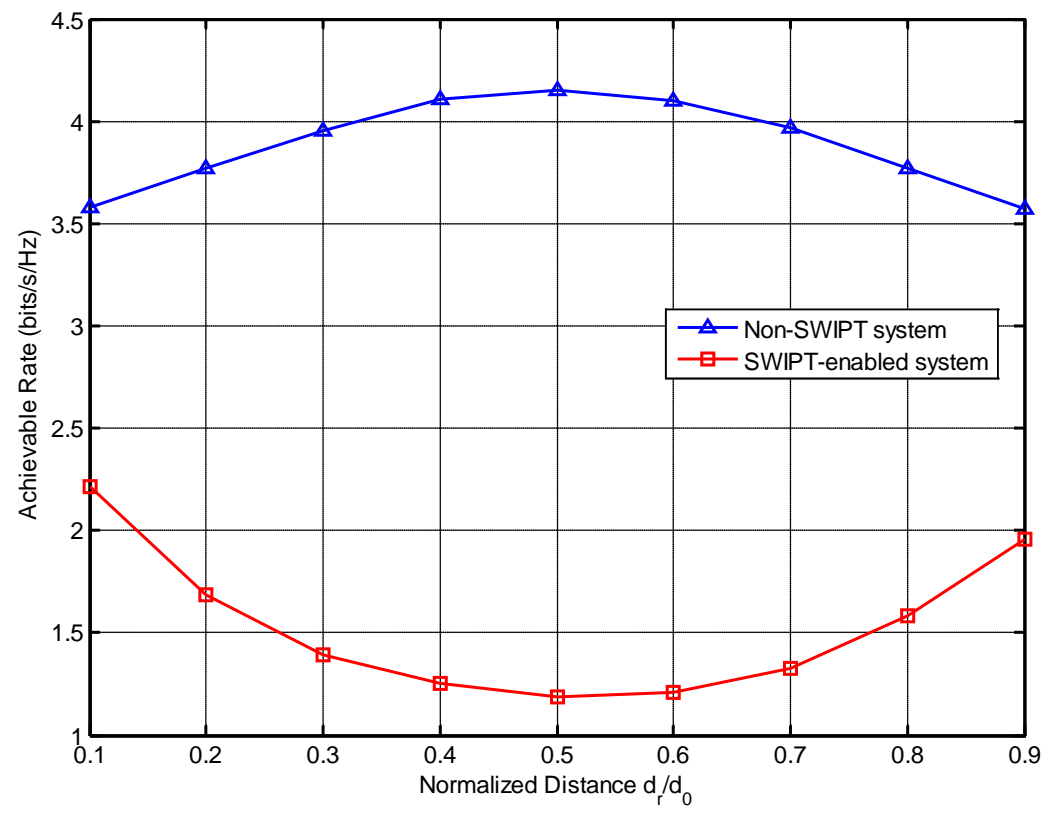

Fig. 4. Comparison of achievable rates of SWIPT-enabled system and non-SWIPT system versus relay position $d_{r} / d_{0}$ with $N=64$ and $P_{S}=30 \mathrm{dBm}$.

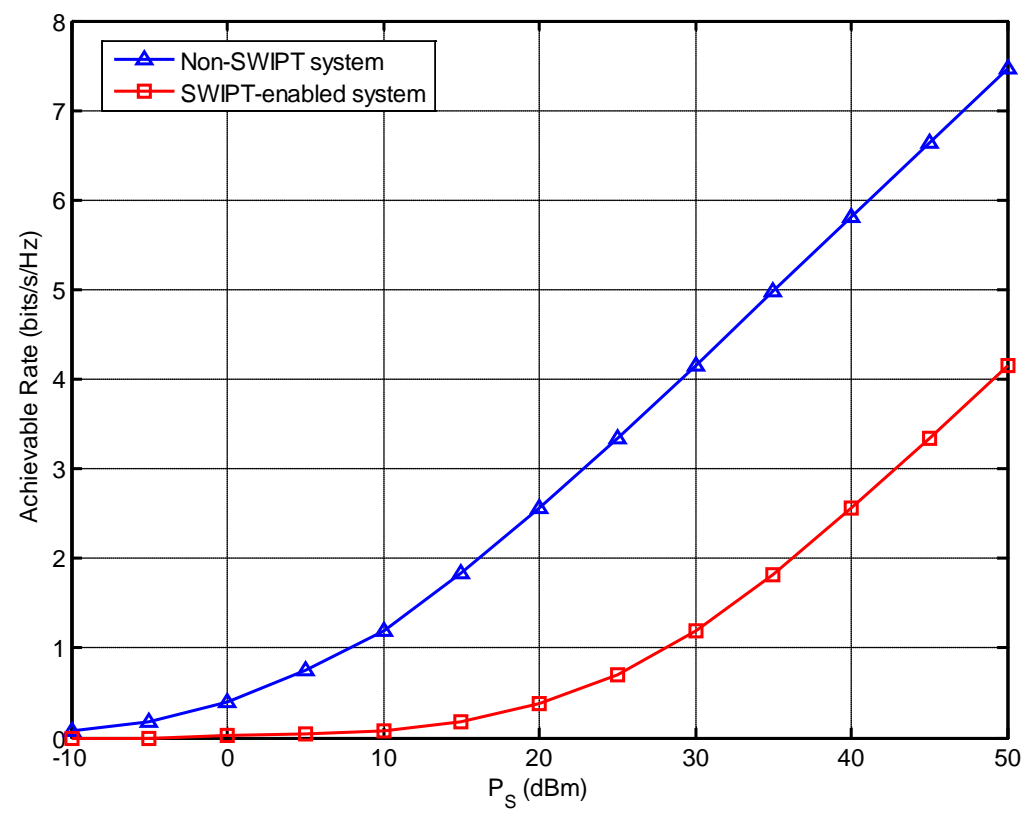

Fig. 5. Comparison of achievable rates of SWIPT-enabled system and non-SWIPT system versus $P_{S}$ with $N=64$ and the relay located at the middle point between the source and its destination.

The achievable information rates of the two systems versus $d_{r} / d_{0}$ and versus $P_{S}$ are respectively plotted in Fig. 4 and Fig.5. From Fig. 4, it can be seen that for the SWIPT-enabled OFDM DF relay system, the closer the relay is placed to the source, the better performance the system can achieve. Such an observation is very different from 
conventional non-SWIPT system, where the maximum achievable information rate is achieved only when the relay node is located at the middle point on the source-destination line.

Moreover, both Fig. 4 and Fig. 5 show that the performance of the non-SWIPT system is superior to that of the SWIPT-enabled system. The reasons may be that, for the SWIPT-enabled system, due to the fading of the source-relay channel, the harvested energy at the relay is very limited. Even though, a part of the harvested energy has to be used for information decoding, leading to less available power at the relay for the information transmission over the second hop, which limits the end-to-end information rate of the SWIPT-enabled system. Moreover, for the SWIPT-enabled system, although the total available power of the system slightly larger than $P_{S}$ due to extra power harvested at the relay, the supplied power $P_{S}$ just can be allocated at the source and but only limited power harvested at the relay can be allocated at the relay. Whereas, for the non-SWIPT system, the available power can be fully allocated between the source and relay nodes to adapt the channel quality of the two links. Comparatively, traditional non-SWIPT system has much larger free space to optimally allocate the power than the SWIPT-enabled system. Therefore, traditional non-SWIPT system achieves better system performance than the SWIPT-enabled system.

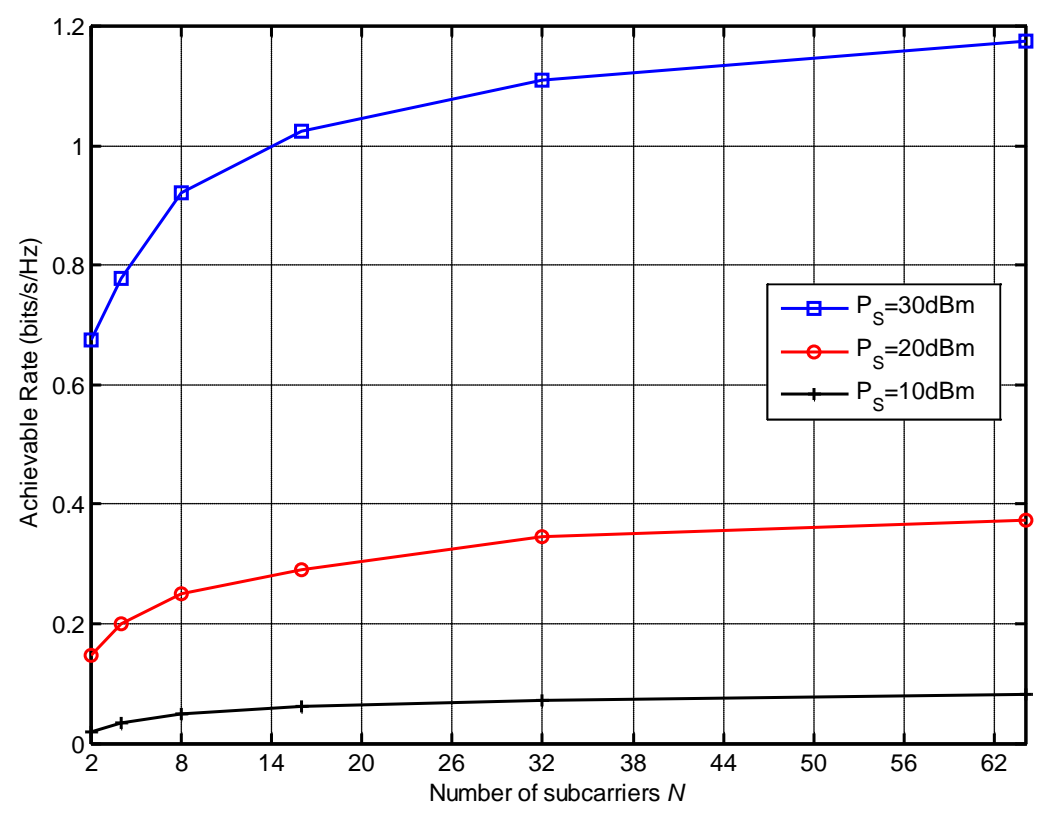

Fig. 6. Achievable rate versus the number of subcarriers $N$ with the relay located at the middle point between the source and destination.

From Fig. 5, one can also see that with the increment of the source power $P_{S}$, the performances of the non-SWIPT system and SWIPT-enabled system increase, and the performance gap between the two systems also increases with the growth of $P_{S}$. This is because that in low power regime, noise is the dominant factor in affecting the system performance, so the impact of PA becomes slight and thus the performance gap between the two scheme is relatively small, while in high power regime, PA becomes the dominant factor rather than the noise, which enlarges the performance gap. As a result, Fig. 5 also indicates that in the high power regime, PA plays a very important role in system performance enhancement, since in the simulations of Fig. 5, both our SWIPT-enabled and traditional 
non-SWIPT system involves the same SP, and in this case, PA becomes the main factor to cause the performance gap.

We also discuss the impact of the number of subcarrier $N$ on the system performance of the SWIPT-enabled OFDM DF relay system. Fig. 6 presents the achievable information rate versus $N$ with different $P_{S}$. It shows that with the growth of $N$, the system achievable information rate becomes higher but its increment becomes smaller.

\section{Conclusion}

In this paper, we investigated the PS based SWIPT for two-hop OFDM DF relay system. To explore the system performance limit, we proposed an efficient RA algorithm to maximize the achievable information rate of the system. With simulations, it was shown that compared with the non-SWIPT system, there is some performance loss in the SWIPT-enabled system. It was also shown that to achieve the best system performance, in the two-hop SWIPT-enabled OFDM DF relay system, the relay should be deployed near the source, while in non-SWIPT system, the relay should be deployed at the middle point between the source and the destination.

\section{References}

[1] A. Sendonaris, E. Erkip and B. Aazhang, "User cooperation diversity - part I: system description," IEEE Trans. Commun., vol. 51, no. 11, pp. 1927-1938, Nov. 2003. Article (CrossRef Link)

[2] J. N. Laneman, D. N. C. Tse and G. W. Wornell, "Cooperative diversity in wireless networks: efficient protocols and outage behavior,” IEEE Trans. Inf. Theory, vol. 50, no. 12, pp. 3062-3080, Dec. 2004. Article (CrossRef Link)

[3] G. Kramer, M. Gastpar and P. Gupta, "Cooperative strategies and capacity theorems for relay networks,” IEEE Trans. Inf. Theory, vol. 51, no. 9, pp. 3037-3063, Sep. 2005. Article (CrossRef Link)

[4] C. Xing, S. Ma, Z. Fei, Y. Wu and H. V. Poor, “A general robust linear transceiver design for multi-hop amplify-and-forward MIMO relaying systems,” IEEE Trans. Signal Process., vol. 61, no. 5, pp. 1196-1209, May 2013. Article (CrossRef Link)

[5] W. Yu and R. Lui, "Dual methods for nonconvex spectrum optimization of multicarrier systems," IEEE Trans. Commun., vol. 54, no. 7, pp. 1310-1322, Jul. 2006. Article (CrossRef Link)

[6] L. Lv and Q. Zhu, "Joint relay selection and resource allocation for cooperative OFDMA network," KSII Trans. Internet Inf. Syst., vol. 6, no. 11, pp. 3008-3025, Nov. 2012. Article (CrossRef Link)

[7] Y. Li, W. Wang, J. Kong, W. Hong, X. Zhang and M. Peng, "Power allocation and subcarrier pairing in OFDM-based relaying networks,” in Proc. of IEEE ICC, pp. 2602-2606, 2008. Article (CrossRef Link)

[8] C-N. Hsu, H-J. Su and P.-H. Lin, "Joint subcarrier pairing and power allocation for OFDM transmission with decode-and-forward relaying,” IEEE Trans. Signal Process., vol. 59, no. 99, pp. 399-414, Jan. 2011. Article (CrossRef Link)

[9] Y. Fu and Q. Zhu, "A joint resource allocation scheme for relay enhanced multi-cell orthogonal frequency division multiple networks,” KSII Trans. Internet Inf. Syst., vol. 7, no. 2, pp. 288-307, Feb. 2013. Article (CrossRef Link)

[10] A. Kansal, J. Hsu, S. Zahedi and M. B. Srivastava, "Power management in energy harvesting sensor networks,” ACM Trans. Embed. Comput. Syst., vol. 6, pp. 1-35, Sept. 2007. Article (CrossRef Link)

[11] L. Varshney, "Transporting information and energy simultaneously," in Proc. of IEEE ISIT, pp. 1612-1616, 2008. Article (CrossRef Link)

[12] P. Grover and A. Sahai, "Shannon meets Tesla: wireless information and power transfer," in Proc. 
of IEEE ISIT, pp. 2363-2367, 2010. Article (CrossRef Link)

[13] R. Zhang and C. K. Ho, "MIMO broadcasting for simultaneous wireless information and power transfer," in Proc. of IEEE GLOBECOM, pp. 1-5, 2011. Article (CrossRef Link)

[14] L. Liu, R. Zhang and K. C. Chua, "Wireless information and power transfer: a dynamic power splitting approach,” IEEE Trans. Commun., vol. 61, no. 9, pp. 3990-4001, Sept. 2013. Article (CrossRef Link)

[15] K. Huang and E. Larsson, "Simultaneous information and power transfer for broadband wireless systems,” IEEE Trans. Signal Process., vol. 61, no. 23, pp. 5972-5986, Dec. 2013. Article (CrossRef Link)

[16] D.W.K. Ng, E.S. Lo and R. Schober, "Wireless information and power transfer: energy efficiency optimization in OFDMA systems," IEEE Trans. Wirel. Commun., vol. 12, no. 12, pp. 6352-6370, Apr. 2014. Article (CrossRef Link)

[17] X. Zhou, R. Zhang and C. K. Ho, "Wireless information and power transfer in multiuser OFDM systems,” IEEE Trans. Wirel. Commun., vol. 13, no. 4, pp. 2282-2294, Dec. 2013. Article (CrossRef Link)

[18] A. A. Nasir, X. Zhou, S. Durrani and R. A. Kennedy, "Relaying protocols for wireless energy harvesting and information processing," IEEE Trans. Wirel. Commun., vol. 12, no. 7, pp. 3622-3636, Jul. 2013. Article (CrossRef Link)

[19] G. Du, K. Xiong, Y. Zhang and Z. Qiu, “Outage analysis and optimization for four-phase two-way transmission with energy harvesting relay,” KSII Trans. Internet Inf. Syst., vol. 8, no. 10, pp. 3321-3341, Oct. 2014. Article (CrossRef Link)

[20] G. Du, K. Xiong, Y. Zhang and Z. Qiu, "Outage analysis and optimization for time switching-based two-way relaying with energy harvesting relay node," KSII Trans. Internet Inf. Syst., vol. 9, no. 2, pp. 545-563, Feb. 2015. Article (CrossRef Link)

[21] G. Du, K. Xiong and Z. Qiu, "Outage analysis of cooperative transmission with energy harvesting relay: time switching versus power splitting,” Math. Probl. Eng., vol. 2015, Article ID 598290, 2015. Article (CrossRef Link)

[22] G. Zheng, Z. Ho, E.A. Jorswieck and B. Ottersten, "Information and energy cooperation in cognitive radio networks,” IEEE Trans. signal Process., vol. 62, no. 9, pp. 2290-2303, Apr. 2014. Article (CrossRef Link)

[23] K. Xiong, P. Fan, C. Zhang and K.B. Letaief, "Wireless information and energy transfer for two-hop non-regenerative MIMO-OFDM relay networks," IEEE J. Sel. Areas Commun., vol. 33, no. 8, pp. 1595-1611, Aug. 2015. Article (CrossRef Link)

[24] R. A. Loodaricheh, S. Mallick and V. K. Bhargava, "Resource allocation for OFDMA systems with selective relaying and energy harvesting," in Proc. of VTC-Fall, 2014. Article (CrossRef Link)

[25] Y. Liu and X. Wang, "Information and energy cooperation in OFDM relaying: protocols and optimization,” IEEE Trans. Veh. Technol., 2015, to appear. Article (CrossRef Link)

[26] M. Hajiaghayi, M. Dong and B. Liang, "Jointly optimal channel pairing and power allocation for multichannel multihop relaying,” IEEE Trans. Signal Process., vol. 59, no. 10, pp. 4998-5012, Oct. 2011. Article (CrossRef Link)

[27] Z. Fang, T. Song and T. Li, "Energy harvesting for two-way OFDM communications under hostile jamming,” IEEE Signal Process. Lett., vol. 22, no. 4, pp. 413-416, Apr. 2015. Article (CrossRef Link)

[28] I. Hammerstrom and A. Wittneben, "On the optimal power allocation for nonregenerative OFDM relay links," in Proc. of IEEE ICC, pp. 4463-4468, 2006. Article (CrossRef Link) 


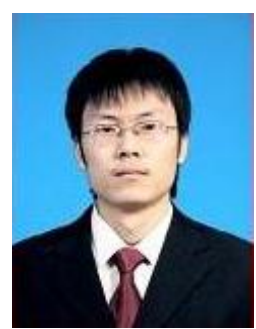

Xiaofei Di received his B.S. degree in Computer Science and Technology from the School of Computer and Information Technology, Beijing Jiaotong University (BJTU), Beijing, China, in 2009. He is currently pursuing for his Ph.D. degree in Signal and Information Processing at the School of Computer and Information Technology, BJTU. His research interests include wireless relay networks, rateless codes, energy efficient and energy harvesting wireless communication.

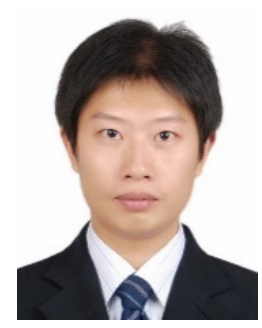

Ke Xiong received the B.S. and Ph.D. degrees from School of Computer and Information Technology, Beijing Jiaotong University (BJTU), Beijing, China, in 2004 and 2010, respectively. From April 2010 to February 2013, he was a Postdoctoral Research Fellow with the Department of Electronic Engineering, Tsinghua University, Beijing. In March 2013, he joined the faculty of School of Computer and Information Technology, BJTU, where he has now become an Associate Professor and also a Ph.D. \& Master's supervisor. He became a member of IEEE in 2014. From Sept. 2015 to Sept. 2016, he is a visiting scholar of University of Maryland, College Park, USA. He has published more than 60 academic papers in referred journals and conferences, including IEEE Journal of Selected Area In Communications (IEEE JSAC), IEEE Transactions on Wireless Communications (IEEE TWC), IEEE Transactions on Signal Rocessing (IEEE TSP), IEEE Transactions on Vehicular Technology (IEEE TVT), IEEE ICC and IEEE GLOBECOM. He was a Session Chair for IEEE GLOBECOM 2012, IET ICWMMN 2013, IEEE ICC 2013, ACM MOMM 2014 and the Publicity and Publication Chair for IEEE HMWC 2014. Prof. Xiong now serves as an Editor of 3 international journals and 2 Chinese journals. He also serves as a reviewer for more than 10 SCI-indexed international leading journals, such as IEEE JSAC, IEEE TWC, IEEE Transactions on Communications (IEEE TCOM), IEEE TVT, IEEE Communication Letters and IET Communications. His current research interests include mobile internet, wireless cooperative networks, wireless network coding, vehicular networks and internet of things.

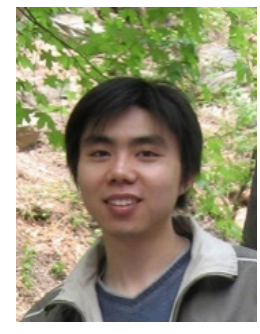

Yu Zhang received his B.S. and Ph.D. degrees from the School of Computer and Information Technology, BJTU, Beijing, R.P. China, in 2004 and 2013, respectively. He is currently a Post Doctor with the School of Computer and Communication Engineering, University of Science and Technology Beijing, China. His research interests include next generation networks, wireless cooperative networks, etc.

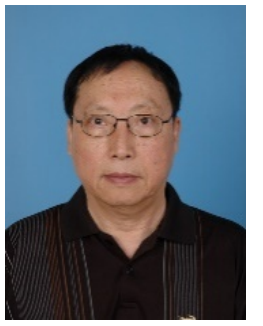

Zhengding Qiu received B.S. degree in Communication Engineering in 1967, and M.S. degree in Digital Signal Processing in 1981 from Northern Jiaotong University, Beijing respectively. During 1967 to 1978, he was a communication engineer at LiuZhou Railway Bureau. Since 1981, he worked at Institute of Information Science, Northern Jiaotong University, where he became a lecturer in 1983, an associate professor in 1987 and a full professor in 1991. During 1999 to 2000, he was a visiting research fellow at Electronic Engineering Lab of Kent University, UK, and engaged in image processing and biometrics processing. From 1993 to 2001, professor Qiu undertook program "Research and Implement of Multimedia Conferences Terminal and System" which is one of Chinese Hi-tech projects and program "the Research and Implement of Multimedia Service Platform over IP network”. His research field includes digital signal processing, multimedia communication and processing and parallel processing. 